\title{
A CONSTRUCTION PRINCIPLE FOR PROPER SCORING RULES
}

\author{
JONAS R. BREHMER
}

(Communicated by Qi-Man Shao)

\begin{abstract}
Proper scoring rules enable decision-theoretically principled comparisons of probabilistic forecasts. New scoring rules can be constructed by identifying the predictive distribution with an element of a parametric family and then applying a known scoring rule. We introduce a condition which ensures propriety in this construction and thereby obtain novel proper scoring rules.
\end{abstract}

\section{INTRODUCTION}

In order to account for the inherent uncertainty of future quantities or events, it is preferable to issue forecasts in the form of probability distributions [6]. One way to measure the predictive ability of such probabilistic forecasts is to assign a score, or loss, $S(F, y)$ to each pair of forecast distribution $F \in \mathcal{F}$ and observation $y \in \mathrm{O}$. In this setting $\mathrm{O}$ is a topological space with Borel $\sigma$-algebra $\mathcal{O}$ and $\mathcal{F}$ is a class of probability distributions on $(\mathrm{O}, \mathcal{O})$. A scoring rule is a function $S: \mathcal{F} \times \mathrm{O} \rightarrow \overline{\mathbb{R}}$ such that for all $F, G \in \mathcal{F}$ the expectation

$$
\mathbb{E}_{G} S(F, Y)=\int_{\mathrm{O}} S(F, y) \mathrm{d} G(y)
$$

is well defined. Here we let $\overline{\mathbb{R}}:=[-\infty, \infty]$ be the extended real line. The scoring rule $S$ is proper relative to $\mathcal{F}$ if

$$
\mathbb{E}_{G} S(G, Y) \leq \mathbb{E}_{G} S(F, Y)
$$

for all $F, G \in \mathcal{F}$. It is strictly proper if equality holds if and only if $F=G$. If a forecaster believes that the quantity $y$ is drawn from the distribution $G$ and receives a penalty $S(F, y)$ for reporting $F$, then propriety ensures that reporting her true belief $F=G$ is an optimal strategy in expectation. For recent reviews of the theory and application of proper scoring rules we refer to [2, 7], and [3].

Various proper scoring rules have been proposed in the literature, in particular for the special situation where each member of $\mathcal{F}$ admits a density with respect to some $\sigma$-finite measure on $(\mathrm{O}, \mathcal{O})$. The logarithmic score $[8$ is defined via

$$
\log S(f, y):=-\log f(y),
$$

where $f$ denotes the probability density function of $F$. It is the most popular strictly proper scoring rule for densities since it connects to various fundamental statistical concepts, such as maximum-likelihood estimation, information criteria,

Received by the editors February 25, 2021, and, in revised form, May 17, 2021, and July 23, 2021.

2020 Mathematics Subject Classification. Primary 62C99.

(C) 2021 by the author under Creative Commons Attribution-Noncommercial 3.0 License (CC BY NC 3.0) 
and Bayes factors [7]. For $\mathrm{O}=\mathbb{R}^{d}$ a popular scoring rule which depends on the first two moments only is the Dawid-Sebastiani $(D S)$ score [4]. If $\mathcal{F}$ is a class of distributions with finite second moments, then it is given by

$$
\operatorname{DSS}(F, y):=\log \operatorname{det} \Sigma_{F}+\left(y-\mu_{F}\right)^{\top} \Sigma_{F}^{-1}\left(y-\mu_{F}\right),
$$

where $\mu_{F}$ and $\Sigma_{F}$ denote the mean and the covariance matrix of the predictive distribution $F$. The DS score is proper, but not strictly proper, as distributions with the same first and second moments attain the same score.

This work is motivated by the fact that, up to unimportant constants, the DS score of $F$ equals the logarithmic score of a multivariate normal distribution with the same mean and covariance matrix as $F$. More precisely,

$$
\begin{aligned}
\operatorname{DSS}(F, y) & =-2 \log \left(\varphi\left(y \mid \mu_{F}, \Sigma_{F}\right)\right)-d \log (2 \pi) \\
& =2 \operatorname{LogS}\left(\varphi\left(\cdot \mid \mu_{F}, \Sigma_{F}\right), y\right)-d \log (2 \pi),
\end{aligned}
$$

where $\varphi(\cdot \mid \mu, \Sigma)$ denotes the density of the multivariate normal distribution with mean $\mu$ and covariance matrix $\Sigma$. This connection raises the question, under which conditions we obtain a proper scoring rule by identifying the predictive distribution $F$ with an element of a parametric family (e.g. the normal distributions) and then applying another proper scoring rule (e.g. the logarithmic score). The Section 2 gives a simple condition which ensures propriety in this construction and is restricted to neither the normal family nor the logarithmic score. The paper concludes with several examples which yield new proper scoring rules and recover existing ones.

\section{Construction PRinciple}

Let $\mathcal{E}:=\left\{F_{\theta} \mid \theta \in \Theta\right\} \subseteq \mathcal{F}$ be a parametric family of distributions with parameter space $\Theta$. Let $\phi: \mathcal{F} \rightarrow \mathcal{E}, F \mapsto F_{\theta}$ be a mapping onto $\mathcal{E}$ and write $\theta_{F}$ for the parameter $\theta$ in $\phi(F)=F_{\theta}$.

Theorem 2.1. Let $S: \mathcal{F} \times \mathrm{O} \rightarrow \overline{\mathbb{R}}$ be a proper scoring rule and $\phi: \mathcal{F} \rightarrow \mathcal{E}$. If there is a function $H: O \rightarrow \mathbb{R}$ which is integrable with respect to all $F \in \mathcal{F}$ and such that for all $F, G \in \mathcal{F}$

$$
\mathbb{E}_{G} S(\phi(F), Y)+\mathbb{E}_{G} H(Y)=\mathbb{E}_{\phi(G)} S(\phi(F), Y)+\mathbb{E}_{\phi(G)} H(Y),
$$

then the scoring rule

$$
S^{*}(F, y)=S(\phi(F), y)=S\left(F_{\theta_{F}}, y\right)
$$

is proper.

Proof. For $F, G \in \mathcal{F}$ invoke Equation (2.1) two times to obtain

$$
\begin{aligned}
\mathbb{E}_{G} S^{*}(F, Y)=\mathbb{E}_{G} S(\phi(F), Y) & =\mathbb{E}_{\phi(G)} S(\phi(F), Y)+\mathbb{E}_{\phi(G)} H(Y)-\mathbb{E}_{G} H(Y) \\
& \geq \mathbb{E}_{\phi(G)} S(\phi(G), Y)+\mathbb{E}_{\phi(G)} H(Y)-\mathbb{E}_{G} H(Y) \\
& =\mathbb{E}_{G} S(\phi(G), Y)=\mathbb{E}_{G} S^{*}(G, Y),
\end{aligned}
$$

where the inequality stems from the propriety of $S$.

Strict propriety in Theorem 2.1 is only possible for special choices of $\mathcal{E}$ and $\phi$, which render the mapping $\phi$ a bijection, since otherwise two different distributions can attain the same score. 
Exponential families are natural and flexible candidates for distributional classes in statistics. We call a set of densities $\{f(\cdot \mid \theta) \mid \theta \in \Theta\}$ on $\mathrm{O}$ an exponential family if any member can be represented via

$$
f(y \mid \theta)=h(y) \exp \left(\eta(\theta)^{\top} t(y)-A(\theta)\right)
$$

for measurable functions $h: \mathrm{O} \rightarrow(0, \infty), t: \mathrm{O} \rightarrow \mathbb{R}^{m}, \eta: \Theta \rightarrow \mathbb{R}^{m}$, and $A: \Theta \rightarrow \mathbb{R}$, where $m \in \mathbb{N}$. The mapping $A$ is often called log-partition function and $t$ is a sufficient statistic for the parameter $\theta$; see [1] for details.

When the scoring rule $S$ in Theorem 2.1 is the logarithmic score, exponential families are convenient candidates for the class $\mathcal{E}$. In detail, let $\mathcal{E}$ be an exponential family on $\mathrm{O}$ and set $H(y):=\log h(y)$, then Equation (2.1) holds if

$$
\mathbb{E}_{G} t(Y)=\mathbb{E}_{\phi(G)} t(Y),
$$

i.e. if the expectations of $t$ agree. Since $\phi(G)$ is a member of the exponential family $\mathcal{E}$, the right-hand side of $(2.2)$ can be calculated and expressed in terms of $\theta \in \Theta$ via the partial derivatives of the log-partition function $A$. If a closed-form expression exists, this yields sufficient conditions on the mapping $\phi: \mathcal{F} \rightarrow \mathcal{E}$ for (2.1) to hold; see Section 3 for concrete examples.

Another possible choice for $S$ in Theorem 2.1 which fits well with exponential families is the Hyvärinen score [9]. Let $\mathrm{O}=\mathbb{R}^{d}$ and let $\nabla$ denote the gradient and $\Delta$ the Laplace operator. Define $\mathcal{L}^{*}$ as the class of densities on $\mathrm{O}$ which are twice differentiable, positive almost everywhere, and such that $\nabla \log (f(y)) g(y) \rightarrow 0$ as $\|y\| \rightarrow \infty$ for all $f, g \in \mathcal{L}^{*}$. Then the Hyvärinen score is given by

$$
\operatorname{HyvS}(f, y):=\Delta \log f(y)+\frac{1}{2}\|\nabla \log f(y)\|^{2}
$$

and it is a strictly proper scoring rule relative to $\mathcal{L}^{*}$ if its expectation is finite. The Hyvärinen score has the remarkable property that it is 0-homogeneous, i.e. to compute $\operatorname{HyvS}(f, y)$ the predictive density $f$ needs to be specified up to the normalization constant only; see [9], [10], and [5] for details.

To connect to Theorem 2.1 assume for simplicity that $\mathcal{E}$ is an exponential family of distributions on $\mathrm{O}=\mathbb{R}^{d}$ where the function $h$ is constant and all densities satisfy the regularity conditions of the class $\mathcal{L}^{*}$. If we define $W_{\theta}(y):=\eta(\theta)^{\top} t(y)$, then the Hyvärinen score on $\mathcal{E}$ is completely determined by

$$
\Delta W_{\theta}(y)=\sum_{i=1}^{m} \eta_{i}(\theta) \Delta t_{i}(y) \quad \text { and } \quad \nabla W_{\theta}(y)=\sum_{i=1}^{m} \eta_{i}(\theta) \nabla t_{i}(y),
$$

where the index $i$ denotes the $i$-th component of a vector in $\mathbb{R}^{m}$. As a consequence, we can set $H=0$ and Equation (2.1) holds if the derivatives of $t$ satisfy

$$
\begin{aligned}
\mathbb{E}_{G} \Delta t_{i}(Y) & =\mathbb{E}_{\phi(G)} \Delta t_{i}(Y), \\
\mathbb{E}_{G}\left[\nabla t_{i}(Y)^{\top} \nabla t_{j}(Y)\right] & =\mathbb{E}_{\phi(G)}\left[\nabla t_{i}(Y)^{\top} \nabla t_{j}(Y)\right],
\end{aligned}
$$

for $i, j=1, \ldots, m$, giving $m+m(m+1) / 2$ identities. Similar to (2.2) these equations provide sufficient conditions for (2.1) to hold, which can be used to define a suitable mapping $\phi: \mathcal{F} \rightarrow \mathcal{E}$ in Theorem 2.1] see Example 3.4. 


\section{ExAmples}

Example 3.1 (Normal family). Let $\mathcal{E}$ consist of the multivariate normal distributions with parameter $\theta=(\mu, \Sigma)$, where $\mu$ is the mean and $\Sigma$ the covariance matrix. The exponential family representation of $\mathcal{E}$ implies $t(y)=\left(y, y y^{\top}\right)$. If $S$ is the logarithmic score, then a mapping $\phi$ can be determined via (2.2). This yields

$$
\left(\mathbb{E}_{G} Y, \mathbb{E}_{G} Y Y^{\top}\right)=\mathbb{E}_{G} t(Y)=\mathbb{E}_{\phi(G)} t(Y)=\left(\mu_{G}, \Sigma_{G}+\mu_{G} \mu_{G}^{\top}\right),
$$

such that $\theta_{F}=\left(\mathbb{E}_{F} Y, \operatorname{Cov}_{F}(Y)\right)$ has to be computed from a predictive distribution $F \in \mathcal{F}$. The resulting scoring function

$$
S^{*}(F, y)=\frac{1}{2}\left(\log \operatorname{det} \Sigma_{F}+d \log (2 \pi)+\left(y-\mu_{F}\right)^{\top} \Sigma_{F}^{-1}\left(y-\mu_{F}\right)\right)
$$

is proper by Theorem 2.1 and an affine transformation of the DS score, as discussed in Section 1 .

Example 3.2 (Laplace family). Let $\mathcal{E}$ be the class of centered Laplace distributions with scale parameter $\nu>0$. Its members have densities $f(y \mid \nu)=$ $(2 \nu)^{-1} \exp (-|y| / \nu)$, thus it forms an exponential family with $t(y)=|y|$. In this situation, (2.2) becomes

$$
\mathbb{E}_{G}|Y|=\mathbb{E}_{G} t(Y)=\mathbb{E}_{\phi(G)} t(Y)=\mathbb{E}_{\phi(G)}|Y|=\nu_{G},
$$

such that $\theta_{F}=\mathbb{E}_{F}|Y|$ is computed from the predictive distribution. Theorem 2.1 implies that the scoring rule

$$
S^{*}(F, y)=\log \left(2 \nu_{F}\right)+\frac{|y|}{\nu_{F}}
$$

where $\nu_{F}=\mathbb{E}_{F}|Y|$ is proper. A natural question is whether it is possible to transfer these arguments to the general class of Laplace distributions with parameters $(\mu, \nu)$, i.e. to the situation of a non-constant location parameter $\mu \in \mathbb{R}$. In this case, (2.1) reads

$$
\mathbb{E}_{G}\left[\frac{\left|Y-\mu_{F}\right|}{\nu_{F}}+H(Y)\right]=\mathbb{E}_{\phi(G)}\left[\frac{\left|Y-\mu_{F}\right|}{\nu_{F}}+H(Y)\right],
$$

with $\theta_{F}=\left(\mu_{F}, \nu_{F}\right)$. Since the random variable $Y$ and the parameter $\mu_{F}$ cannot be separated, it is not clear how to obtain a mapping $\phi$ which satisfies this identity for all $F, G \in \mathcal{F}$ if $\mathcal{F}$ is sufficiently large. Consequently, it is not obvious whether Theorem 2.1 can be applied to the logarithmic score in concert with the general Laplace family.

Example 3.3 (Poisson family). Let $\mathrm{O}=\mathbb{N}$ and $\mathcal{E}$ be the class of Poisson distributions with parameter $\lambda>0$. The exponential family representation implies $t(y)=y$ and (2.2) becomes

$$
\mathbb{E}_{G} Y=\mathbb{E}_{G} t(Y)=\mathbb{E}_{\phi(G)} t(Y)=\mathbb{E}_{\phi(G)} Y=\lambda_{G},
$$

hence $\theta_{F}=\mathbb{E}_{F} Y$ gives a suitable mapping $\phi$. By Theorem 2.1 the resulting scoring rule

$$
S^{*}(F, y)=-y \log \left(\lambda_{F}\right)+\lambda_{F}+\log (y !)
$$

where $\lambda_{F}$ is the expectation of $F$, is proper. 
Example 3.4 (Normal family, continued). Let $\mathcal{E}$ be as in Example 3.1. If $S$ is the Hyvärinen score, the conditions in (2.3) and (2.4) simplify to equations which contain the moments $\mathbb{E}_{G} Y_{i}$ and mixed moments $\mathbb{E}_{G} Y_{i} Y_{j}$ for $i, j=1, \ldots, d$, only. Hence, the mapping $\phi$ of Example 3.1 which is given by the parameter choice $\theta_{F}=\left(\mathbb{E}_{F} Y, \operatorname{Cov}_{F}(Y)\right)$, satisfies these conditions. As a result we obtain a DawidSebastiani type scoring rule given by

$$
S^{*}(F, y)=-2 \operatorname{tr} \Sigma_{F}^{-1}+\left\|\Sigma_{F}^{-1}\left(y-\mu_{F}\right)\right\|^{2}=-2 \operatorname{tr} \Sigma_{F}^{-1}+\left(y-\mu_{F}\right)^{\top} \Sigma_{F}^{-2}\left(y-\mu_{F}\right),
$$

which is proper by Theorem 2.1. It already appears in [9, Section 3.1] in the context of score matching, however, our derivation establishes propriety in wide generality, not only relative to the normal family.

\section{ACKNOWLEDGMENTS}

The author is grateful for funding by the Klaus Tschira Foundation and for infrastructural support provided by the University of Mannheim. I thank Tilmann Gneiting for fruitful comments and discussions and two anonymous referees for helpful suggestions.

\section{RefERENCES}

[1] O. Barndorff-Nielsen, Information and exponential families in statistical theory, Wiley Series in Probability and Statistics, John Wiley \& Sons, Ltd., Chichester, 2014. Reprint of the 1978 original [MR0489333], DOI 10.1002/9781118857281. MR3221776

[2] A. P. Dawid, The geometry of proper scoring rules, Ann. Inst. Statist. Math. 59 (2007), no. 1, 77-93, DOI 10.1007/s10463-006-0099-8. MR2396033

[3] Alexander Philip Dawid and Monica Musio, Theory and applications of proper scoring rules, Metron 72 (2014), no. 2, 169-183, DOI 10.1007/s40300-014-0039-y. MR.3233147

[4] A. Philip Dawid and Paola Sebastiani, Coherent dispersion criteria for optimal experimental design, Ann. Statist. 27 (1999), no. 1, 65-81, DOI 10.1214/aos/1018031101. MR1701101

[5] Werner Ehm and Tilmann Gneiting, Local proper scoring rules of order two, Ann. Statist. 40 (2012), no. 1, 609-637, DOI 10.1214/12-AOS973. MR3014319

[6] Tilmann Gneiting and Matthias Katzfuss, Probabilistic forecasting, Ann. Rev. Stat. Appl. 1 (2014), 125-151.

[7] Tilmann Gneiting and Adrian E. Raftery, Strictly proper scoring rules, prediction, and estimation, J. Amer. Statist. Assoc. 102 (2007), no. 477, 359-378, DOI 10.1198/016214506000001437. MR2345548

[8] I. J. Good, Rational decisions, J. Roy. Statist. Soc. Ser. B 14 (1952), 107-114. MR77033

[9] Aapo Hyvärinen, Estimation of non-normalized statistical models by score matching, J. Mach. Learn. Res. 6 (2005), 695-709. MR2249836

[10] Matthew Parry, A. Philip Dawid, and Steffen Lauritzen, Proper local scoring rules, Ann. Statist. 40 (2012), no. 1, 561-592, DOI 10.1214/12-AOS971. MR3014317

Computational Statistics Group, Heidelberg Institute for Theoretical Studies, Schloss-Wolfsbrunnenweg 35, 69118 Heidelberg, Germany

Email address: Jonas.Brehmer@h-its.org 